\title{
Verzeichnis der für die Literaturnachweise benutzten Abkürzungen.
}

A. = Liebias Annalen der Chemie.

A. ch. = Annales de chimie et de physique.

Am. = American Chemical Journal.

Am. Soc. $=$ Journal of the American Chemical Society.

A. Pth. = Archiv für experimentelle Pathologie und Pharmakologie.

Ar. = Archiv der Pharmazie.

B. = Berichte der Deutsehen Chemischen Gesellschaft.

Bio. Z. = Biochemische Zeitschrift.

Bl. = Bulletin de la Société Chimique de France.

B. $\mathrm{Ph} . \mathrm{P}_{\text {. }}=$ Beiträge zur chemischen $\mathrm{Physiologie}$ und Pathologie.

C. $=$ Chemisches Zentralblatt.

C. $r$. = Comptes rendus des séances de l'Acsdémie des sciences.

Ch. I. = Chemische Industrie.

Ch. Z. = Chemiker-Zeitung.

Chem. N. = Chemical News.

D. = Dinalers polytechn. Journal.

D. R. P. = Patentschrift des Deutschen Reiches.

El. Ch. Z. = Elektrochemische Zeitschrift.

Fr. = (Fresenros') Zeitschrift für analytische Chemie.

Frdl. = Friedränder s Fortschritte der Teerfarbenfabrikation (Berlin, Sprinaer).

G. = Gazetta chimica italians.

H. = (HOPPE-SkYLers) Zeitschrift für physiologische Chemie.

J. $=$ Jabresbericht der Chemie.

J. pr. = Journal für praktische Chemie.

L. V. St. = Landwirtschaftliche Versuchsstationen.

M. = Monatshefte der Chemie.

P. C. H. = Pharmazeutische Zentralballe.

P. Ch. S, = Proceedings of the Chemical Society.

$\mathrm{Ph}$. Ch. = Zeitschrift für physikalische Chemie.

R. = Recueil des travaux chimiques des Pays-Bas.

R. A. L. = Atti della Reale Accademia dei Lincei (Rendiconti).

Soc. $=$ Journal of the Chemical Society of London.

W. = Wiedemans 8 Annalen der Physik.

Z. = Zeitschrift für Chemie.

Z. a. Ch. = Zeitschrift für anorganische Chemie.

Z. Ang. = Zeitschrift für angewandte Chemie.

Z. B. = Zeitschrift für Biologie.

Z. El. Ch. = Zeitschrift für Elektrochemie.

Z. Kr. = Zeitschrift für Krystallographie. 